\title{
MONITORING AND CONTROL OF GRAIN STORAGE USING PLC
}

\author{
K.Kalaivani ${ }^{1}$, V.Anjalipriya ${ }^{2}$, P.Surendar ${ }^{3}$ \\ 1, 2, ${ }^{3}$ Department of Electronics and Instrumentation Engineering, Easwari Engineering College \\ kvani2007@gmail.com,anjilipriya@gmail.com, surendar1990@gmail.com
}

\begin{abstract}
Grain storage occupies a vital role in the economies of developed and developing countries. Proper monitoring of grain storage is essential to reduce grain loss. The present system involves human effort in most of the activities which reduces work efficiency and increases time consumption. These difficulties can be avoided by our proposed project. In this project, the controlling and monitoring of the grain storage area is fully automated using PLC and SCADA. The main objective of the proposed project is to control and maintain the temperature in storage area which prevents the formation of microorganisms and spoilage of grains, to measure the weight using a load cell placed under the conveyor belt and to direct the different sizes of bags to their respective storage places and to detect and control the pests, rats using ultrasound technique. The main parameter that is essential for proper storage of grains is temperature which is taken as input parameter to be controlled using PLC and is measured using a temperature sensor. The SCADA system acquires the required data and monitors the overall process.
\end{abstract}

\section{INTRODUCTION}

We live in a world where everything can be controlled and operated automatically, but there are still few important sectors in our country where automation has not been adopted or not been put to a full- fledged use, perhaps because of several reasons one such reason is cost. Agriculture has been one of the primary occupations of man since early civilizations and even today manual interventions in farming and storing grains are inevitable. In most countries grains are among the most important staple foods. However they are produced on a seasonal basis, and in many places there is only one harvest a year, which itself may be subject to failure. This means that in order to feed the world's population, most of the global production of maize, wheat, rice and millet must be held in storage for periods varying from one month up to more than a year. Grain storage therefore occupies a vital place in the economies of developed and developing countries alike.

India is one of the largest grain producers in the world. It produces 200 million MT (Metric tons) of wheat and rice annually. Seventy-five percent of total grain loss occurs at the farm level. The majority of farm level losses are driven by inadequate storage (22\%), drying of crops $(15 \%)$, transportation $(12 \%)$, and threshing $(10 \%)$ while the rest of the loss scatters among several activities.

\section{CONCEPT OF GRAIN STORAGE}

Grain storage facilities take many forms depending on the quantity of grain to be stored, the purpose of storage, and the location of the store. The stored grain should be assumed as raw material not only as a commodity. The grain storage chain is an essential element for incrementing the agricultural production and to preserve them in perfect technical conditions and redistribute them later. More grains are destroyed because of improper storage

The requirements for a good storage system include:

- Prevention of moisture re-entering the grain after drying

- Protection from insects

- Ease of loading and unloading

- Efficient use of space

- Ease of maintenance and management

\section{EXISTING SYSTEM}

One option is to store bags of grain on husks and then cover the pile with husks. A second option involves digging a pit, and storing bagged or loose grain covered with some husks, mud or sacks. The third option involves building a small cement storage facility. Automated grain storage systems are also in practice in few places which are controlled and monitored by personal computers.

\section{DRAWBACKS OF EXISTING SYSTEMS}

The automated grain storage system existing is based on personal computers. The major difficulties faced in such systems involve programming and time consuming. Loading and unloading of grains also needs human effort, less reliability and flexibility. It is tedious to troubleshoot and communication capabilities are less.

\section{PROPOSED SYSTEM}

In this project, the controlling and monitoring of the grain storage area is fully automated using PLC and SCADA. The main objective of the proposed project is to control and maintain the temperature in storage area which prevents the formation of microorganisms and spoilage of grains, to measure 
the weight using a load cell placed under the conveyor belt and to direct the different sizes of bags to their respective storage places and to detect and control the pests, rats using ultrasound technique.

The main parameter that is essential for proper storage of grains is temperature which is taken as input parameter to be controlled using PLC and is measured using a temperature sensor. The SCADA system acquires the required data and monitors the overall process.

\section{ADVANTAGES OF PROPOSED SYSTEM}

The difficulties that are faced in existing systems in controlling and monitoring of grain storage could be reduced by the use of Programmable Logic Controllers. A conveyor belt is used for loading and unloading of grains which reduces manual effort thereby increasing the effectiveness. The enhanced features of PLC overcome such difficulties and meet the industrial standards. The main advantages are as follows:

- Increased reliability

- Easy programming and more flexibility in modifying the program

- Easy to troubleshoot

- Workload is reduced and the efficiency of storage can be reached

- Faster response time and the grains could be monitored easily.

\section{PROGRAMMABLE LOGIC CONTROLLER}

A Programmable Logic Controller is a digital computer which performs the control logic, sequencing, timing, arithmetic data manipulation and counting functions. Unlike general-purpose computers, the PLC is designed for multiple inputs and output arrangements, extended temperature ranges, immunity to electrical noise, and resistance to vibration and impact. CPU is the brain of the PLC,it consists of microprocessor for implementing the logic and for controlling the communication among the modules. Memory unit is used for storing results of logical operations performed by the processor. IO section consists of input modules and output modules. This system forms the interface by which field devices are connected to the controller. The programming device enters the desired program into the memory of the processor. The power supply supplies DC power to the modules. Ladder logic is the programming language that is primarily used to develop software for programmable logic controllers (PLCs). It represents a program by a graphical diagram based on the circuit diagrams of relay logic hardware. PLC used is OMRON PLC.

\section{SCADA}

SCADA stands for supervisory control and data acquisition. The term SCADA usually refers to centralized systems which monitor and control entire sites, or complexes of systems spread out over large areas. A human machine interface or HMI is the apparatus which presents process data to a human operator, and through which the human operator controls the process. An HMI is usually linked to the SCADA system's databases and software programs, to provide trending, diagnostic data, and management information such as scheduled maintenance procedures, logistic information, detailed schematics for a particular sensor or machine, and expert-system troubleshooting guides. SCADA software used is Intouchwonderware.

\section{METHODOLOGY}

The main operation of storing the grains is carried out through PLC which is used as the controller and interfaced using SCADA for monitoring the process. The required conditions for grains to store are studied thoroughly and the necessary temperature is maintained for the grains. The PLC is programmed using Ladder logic programming language.

The sensors used here are temperature sensor and IR sensor. The input parameter temperature is sensed by the temperature sensor (LM35) and is given as input signal to the PLC. The PLC obtains this value through an analog input module and compares it with the set point mentioned in the program and decides the control action to be taken. If the value is lower than the set point, then PLC actuates the heater.

A conveyor belt is used for loading and unloading of grain bags. An IR sensor is placed at the start point of the conveyor belt. A Load cell is placed under the conveyor belt to measure the weight of grain bags and a mechanical separator is used for directing the grain bags based on their sizes to their respective storage places. When a bag is placed on the belt, the IR sensor at the starting point senses it and the motor is switched ON thereby the conveyor moves. When a load is applied on the load cell, the output is generated which is given to the instrumentation amplifier. The instrumentation amplifier acts as a signal conditioning unit and amplifies the output of the load cell. This output is given to the PLC which in turn drives the gear motor. This operates the mechanical separator which directs the grain bags of different weights to their respective storage places. IR sensors are placed at the unloading side of the conveyor belt in order to detect the grain bags, so that the motor is switched OFF and the conveyor belt stops moving.

The detection and control process of rats and pests is done using ultrasonic sound technique. An IR sensor pair is used to detect the presence of rats and pests in the storage area. An ultrasonic sound (sound waves with a frequency above the upper limit of human hearing) is produced to control the occurrence of rats and pests. NE555 IC is used for this purpose. Ultrasonic sound wave can be generated using electronic circuit. 
SCADA (InTouchWonderware) is used for interfacing with PLC for data gathering and monitoring, thus making the automation process easier.

\section{CONVEYOR SETUP}

A conveyor belt is used for loading and unloading of grain bags to their respective storage places. This conveyor setup consists of a load cell which is placed under the conveyor for measuring the weight of the grain bags. It also consists of a mechanical separator which differentiates the different sizes of grain bags according to their weight and directs them to their respective storage places.

Two IR sensors are placed at the start and end positions of the conveyor belt. When the IR sensor at the start position detects the grain bag, the motor is switched $\mathrm{ON}$ and the conveyor starts moving. Similarly, when the IR sensor placed at the end position detects the grain bag, the motor is switched OFF and the conveyor belt is stopped until the grain bags are unloaded to their respective places.

A load cell is a transducer that is used to convert a force into electrical signal. This conversion is indirect and happens in two stages. Through a mechanical arrangement, the force being sensed deforms a strain gauge. The strain gauge measures the deformation (strain) as an electrical signal, because the strain changes the effective electrical resistance of the wire. The electrical signal output is typically in the order of a few millivolts and requires amplification by an instrumentation amplifier before it can be used. Here the load cell is used for weight measurement. It can measure upto $8 \mathrm{kgs}$ and has four terminals namely input positive, input negative, output positive, output negative. A supply voltage of $5 \mathrm{~V}$ is given to the input positive of load cell and the input negative terminal is grounded. Output of the load cell in millivolts is given to input of instrumentation amplifier for signal conditioning.

When a grain bag is placed on the conveyor belt, an IR sensor detects the grain bag. The output of the IR sensor is below $5 \mathrm{~V}$, so a driver circuit is used. Driver circuit is made with the help of ULN2803 IC. ULN2803 is an Integrated Circuit (IC) chip with a High Voltage/High Current Darlington Transistor Array. The chip takes low level signals (less than $5 \mathrm{~V}$ ) as input and acts as a relay by switching on or off a higher level signal (12V) on the opposite side. Since the output of the IR sensor is below $5 \mathrm{~V}$, a driver circuit is used to give the corresponding value $(12 \mathrm{~V})$ as input to PLC. The output of the PLC is given to relay for switching operation. The relay switch closes, the gear motor starts running and the conveyor belt moves.

Load cell is placed under the conveyor belt to measure the weight of grain bags. When a load is applied on the load cell, the output is generated which is given to the instrumentation amplifier. The instrumentation amplifier acts as a signal conditioning unit and amplifies the output of the load cell. This output is given to the PLC which in turn drives the gear motor.
This operates the mechanical separator which directs the grain bags of different weights to their respective storage places.

\section{TEMPERATURE CONTROL LOOP}

In the process of grain storage, temperature and humidity are the two main factors that affect the grain quality. Insect, mite, fungal and mycotoxin development are controlled by temperature. At temperatures found in grain stores, biological activity of insects, mites, fungi and grain itself, doubles for every $10^{\circ} \mathrm{C}$ rise in temperature.

Traditional methods of on-farm storage generally rely on controlling the moisture content, and to a certain extent the temperature, of the grain going into storage. Without proper conditioning facilities it is unwise to attempt to store the grain for longer than 5-8 weeks.

To accurately control process temperature without extensive operator involvement, a temperature control system relies upon a controller, which accepts a temperature sensor such as a thermocouple or RTD as input. It compares the actual temperature to the desired control temperature, or setpoint, and provides an output to a control element. The controller is one part of the entire control system, and the whole system should be analyzed in selecting the proper controller. The following items should be considered when selecting a controller:

1. Type of input sensor (thermocouple, RTD) and temperature range

2. Type of output required (electromechanical relay, SSR, analog output)

3. Control algorithm needed (on/off, proportional, PID)

4. Number and type of outputs (heat, cool, alarm, limit).

For heating control, the output is on when the temperature is below the setpoint, and off above setpoint. Since the temperature crosses the setpoint to change the output state, the process temperature will be cycling continually, going from below setpoint to above, and back below. In cases where this cycling occurs rapidly, and to prevent damage to contactors and valves, an on-off differential, or "hysteresis," is added to the controller operations. This differential requires that the temperature exceed setpoint by a certain amount before the output will turn off or on again. On-off differential prevents the output from "chattering" or making fast, continual switches if the cycling above and below the setpoint occurs very rapidly.

Table 1: Effect of different temperatures on pests

\begin{tabular}{|c|c|}
\hline TEMPERATURE & EFFECT ON PESTS \\
\hline 30 & Maximum development rate \\
\hline 35 & Development slows \\
\hline 45 & Development stops \\
\hline 50 & Death in hours \\
\hline 60 & Death in minutes \\
\hline
\end{tabular}


The temperature inside the storage area is measured using temperature sensor, LM35. The output of the sensor is given to the PLC and is compared with the set point value of the PI controller. The values are compared and the error is calculated. The analog output from the controller is converted into TPO(Time Proportional Output). The heater coil is switched $\mathrm{ON}$ and $\mathrm{OFF}$ at the required rate and the heating is controlled to reach the set point. If the temperature exceeds set point, the heater coil temperature is reduced to control overheating. A blower is used to suck the air from outside and this air is heated by the heater in order to maintain the temperature in the storage area.

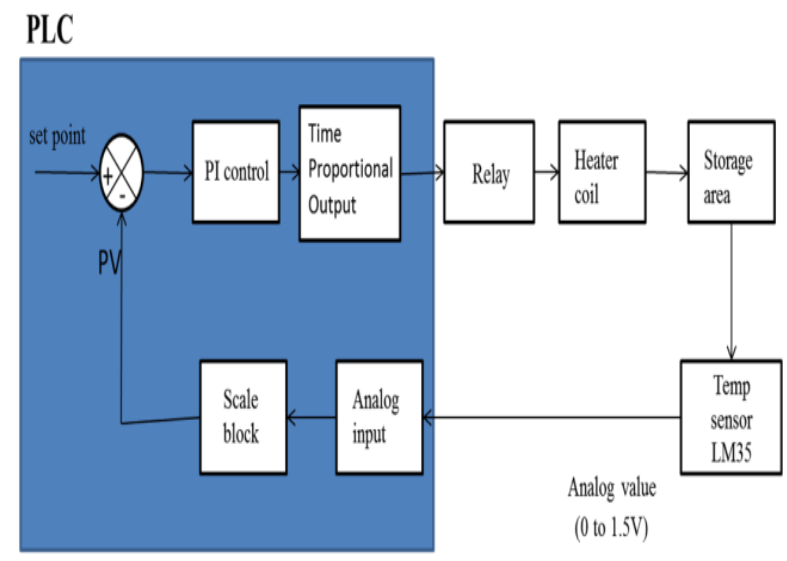

Figure 1: Temperature control loop

\section{RAT DETECTION AND CONTROL}

Ultrasound is cyclic sound pressure with a frequency greater than the upper limit of human hearing. Ultrasound is thus not separated from "normal" (audible) sound based on differences in physical properties, only the fact that humans cannot hear it. The upper frequency limit in humans (approximately $20 \mathrm{kHz}$ ) is due to limitations of the middle ear, which acts as a low-pass filter. Ultrasonic hearing can occur if ultrasound is fed directly into the skull bone and reaches the cochlea through bone conduction without passing through the middle ear. Many animals - such as dogs, cats, dolphins, bats, and mice-have an upper frequency limit that is higher than that of the human ear and thus can hear ultrasound. The use of ultrasonic devices in pest control would be a potential source of environmental friendly technology because ultrasound is clean and nonchemical. Rodents can hear the sound that humans cannot. They can produce and hear ultrasound (sound waves with a frequency above the upper limit of human hearing). The ultrasonic devices for rodent control are practically inaudible to humans with their output frequencies above 20 kilohertz $(\mathrm{kHz})$. They produce ultrasonic noise so aversive to rodents that it drives rodents away.

Ultrasonic sound wave can be generated using electronic circuit. This simple electronic circuit can generate an ultrasonic wave with frequency range of 36 to $72 \mathrm{kHz}$. It is very easy and quick to make this circuit. This circuit uses NE555 IC timer to drive a speaker. If a moving coil tweeter is used, then it is recommended to add a decoupling capacitor around $1 \mathrm{uF}$ $2.2 \mathrm{uF} / 16 \mathrm{~V}$ to block the DC voltage. For adding a circuit safety, a $1 \mathrm{k}$ resistor is inserted between $\mathrm{R} 2$ and the positive supply line, since the pin 7 of 555 IC will be internally shorted to ground at the discharging cycle.

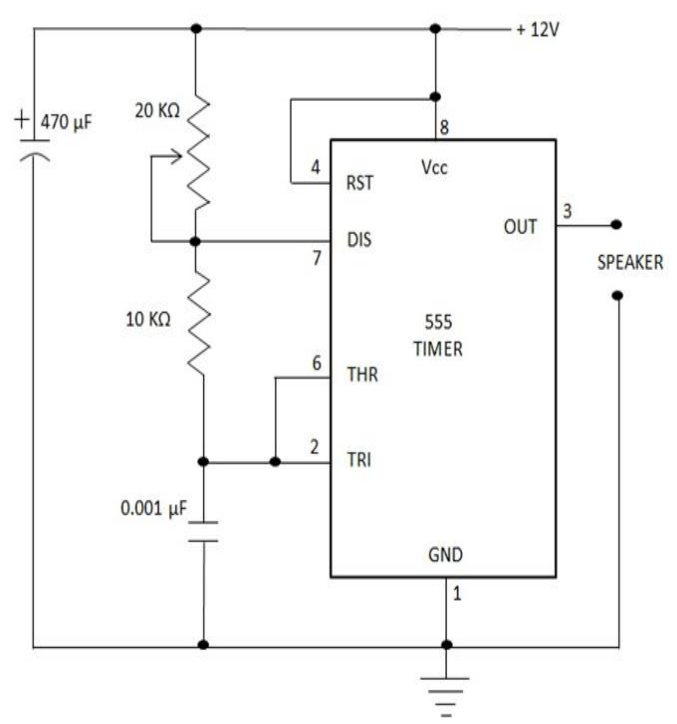

Figure 2: Ultrasonic sound wave generator

\section{DESIGN CALCULATION}

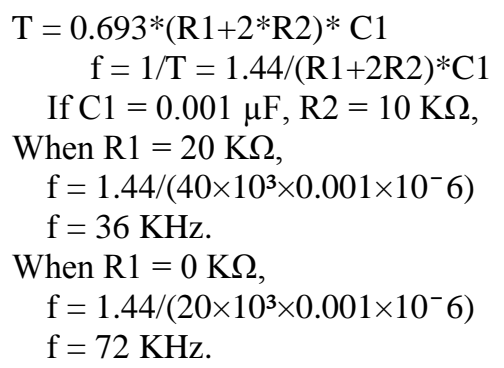

Hence frequency range is $36 \mathrm{KHz}$ to $72 \mathrm{KHz}$

\section{PROCESS SETUP}

The main operation of storing the grains is carried out through PLC which is used as the controller and interfaced using SCADA for monitoring the process. The required conditions for grains to store are studied thoroughly and the necessary temperature is maintained for the grains. The PLC is programmed using Ladder logic programming language.

In this project, the controlling and monitoring of the grain storage area is automated using PLC and SCADA. The main objective of the proposed project is to control and maintain the temperature in storage area which prevents the formation of 
microorganisms and spoilage of grains, to measure the weight using a load cell placed under the conveyor belt and to direct the different sizes of bags to their respective storage places and to detect and control the pests, rats using ultrasound technique.

The main parameter that is essential for proper storage of grains is temperature which is taken as input parameter to be controlled using PLC and is measured using a temperature sensor. The SCADA system acquires the required data and monitor sthe overall process

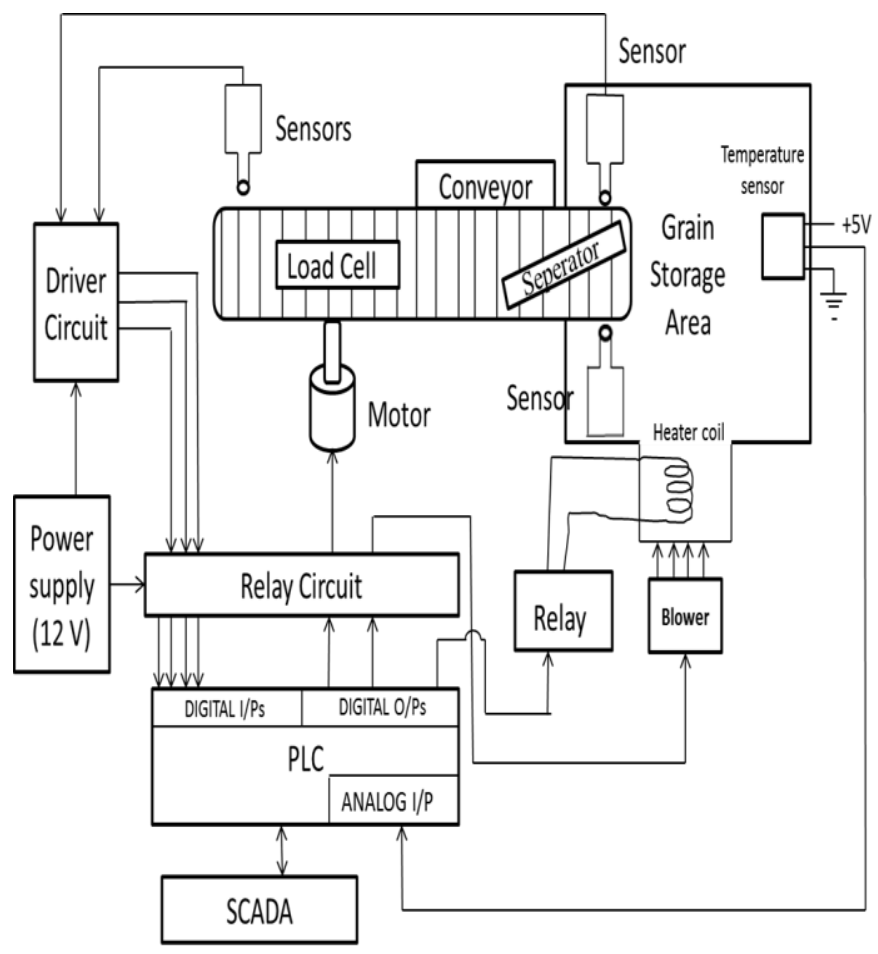

Figure 3: Block diagram of final setup

\section{SIGNAL CONDITIONING OF LOAD CELL}

The output of the load cell is given to an instrumentation amplifier (INA122) in order to amplify the measured value. The output side of the instrumentation amplifier consists of a resistor and a capacitor of values $10 \mathrm{~K} \Omega$ and $100 \mu \mathrm{F}$ respectively. The amplified output is the voltage drop across the $10 \mathrm{~K} \Omega$ resistor which is given to the PLC to drive the gear motor. The $100 \mu \mathrm{F}$ capacitor is used as a filter to reduce the noise and eliminate the unwanted signals.

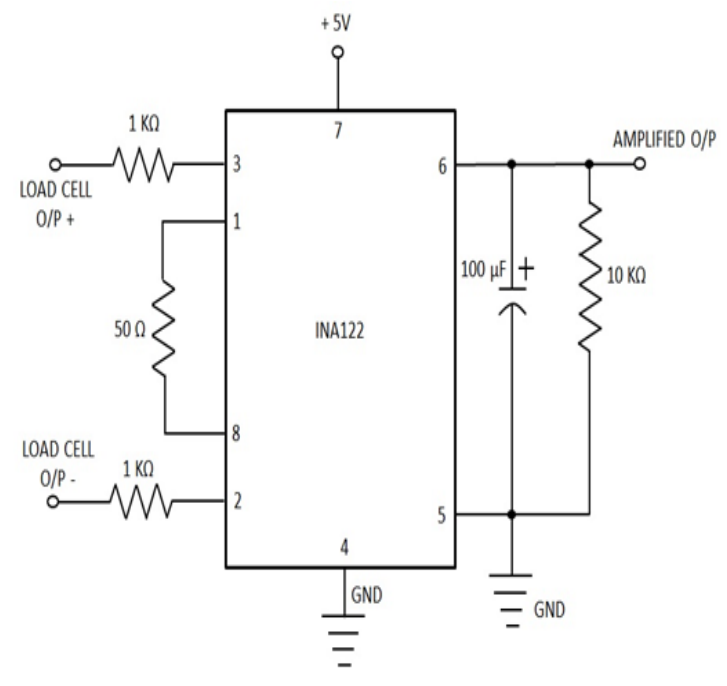

Figure 4: Signal conditioning of load cell

\section{CALCULATION OF GAIN}

The Gain of the amplifier can be set externally by using a resistance $\mathrm{Rg}$.

$$
\text { Gain, } \mathrm{G}=5+(20 \mathrm{k} / \mathrm{Rg})
$$

WhenRg $=50 \Omega$,

Gain, $\mathrm{G}=5+(20 \mathrm{k} / 50)$

Gain, $\mathrm{G}=405$

\section{TEMPERATURE SENSOR}

The measurement of the temperature adopts LM35 module, which is a temperature sensor with output in direction proportion to the centigrade temperature. The temperature range of this sensor is $-55^{\circ} \mathrm{C}$ to $+150^{\circ} \mathrm{C}$.

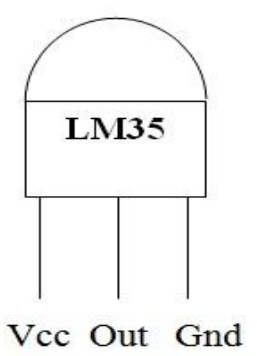

Figure 5: Temperature sensor LM35

\section{IR SENSOR}

The basic idea is to send infrared light through IR-LEDs, which is then reflected by any object in front of the sensor. An infrared sensor is an electronic device that emits and/or detects infrared radiation in order to sense some aspect of its surroundings. Infrared sensors can measure the heat of an 
object, as well as detect motion. Many of these types of sensors only measure infrared radiation, rather than emitting it, and thus are known as passive infrared (PIR) sensors.

All objects emit some form of thermal radiation, usually in the infrared spectrum. This radiation is invisible to our eyes, but can be detected by an infrared sensor that accepts and interprets it. In a typical infrared sensor like a motion detector, radiation enters the front and reaches the sensor itself at the center of the device.

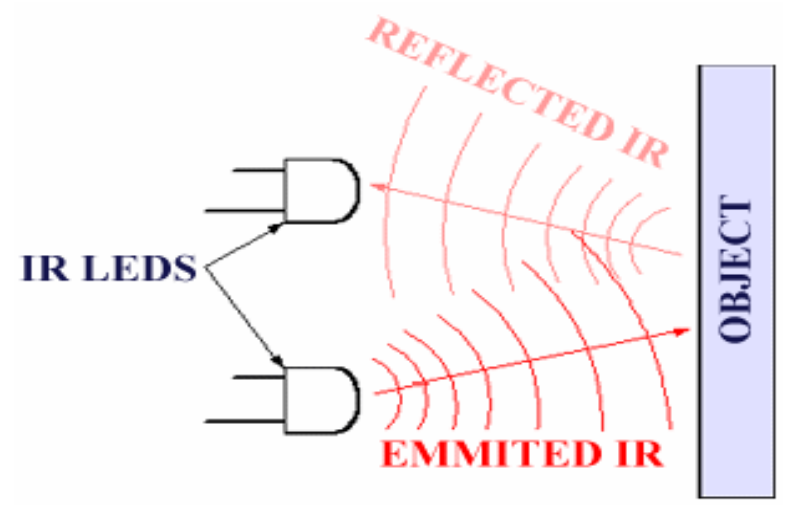

Figure 6: IR sensor working principle

\section{INSTRUMENTATION AMPLIFIER}

An instrumentation amplifier is a type of differential amplifier that has been outfitted with input buffers, which eliminate the need for input impedance matching and thus make the amplifier particularly suitable for use in measurement and test equipment.

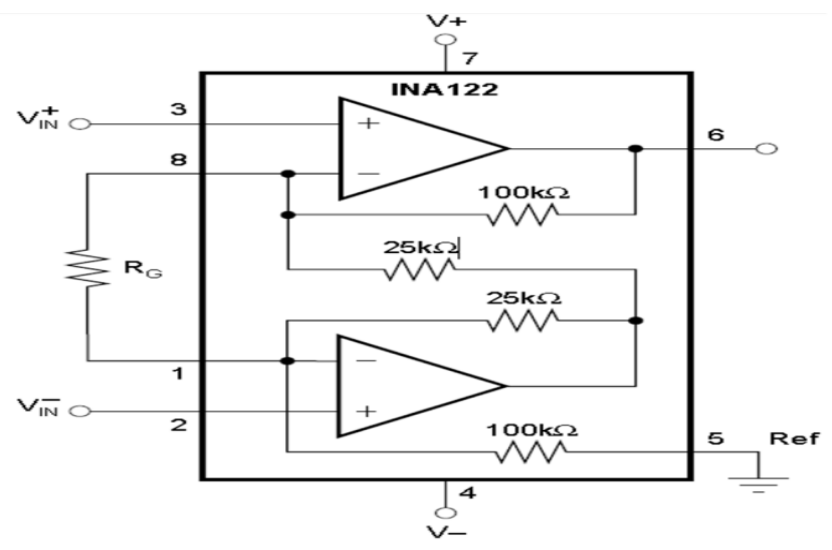

Figure 7: Instrumentation Amplifier

\section{SCADA SCREEN}

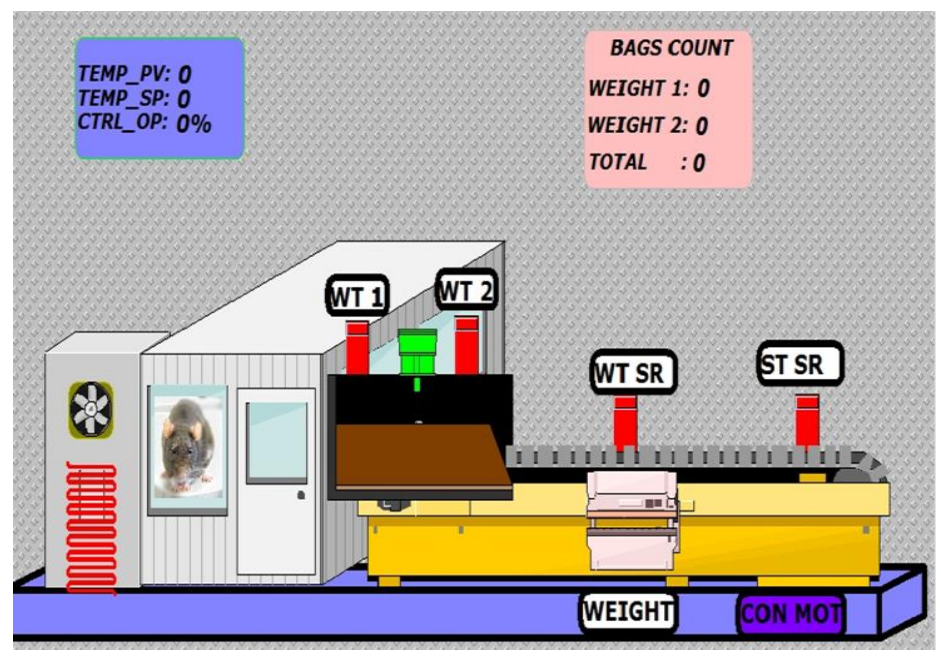

\section{CONCLUSIONS}

The grain storage technique is of great importance in India in future and to globalise our standards in agriculture. The technology in India has to be enhanced. Our aim was to utilize the learnt instrumentation principles and put that into use so that it will improve the standards of our nation. India still has to improve its automation techniques especially in the field of agriculture. We used our knowledge in programmable logic controllers and wanted to use it for a cause that would help in the betterment of our nation. We made research on different grain storage techniques to know the desiarable conditions for proper storage, and then we decided the parameters to be mainly monitored and the required ways of controlling them.

The loading and unloading of grain bags in the grain storage area is done by the use of a conveyor belt. A load cell is placed under the conveyor belt to measure the weight of the grain bags and also to have a count on total number of bags in the storage area. A mechanical separator directs the grain bags to their respective storage places based to their sizes. This reduces human effort and also saves time. The temperature in the grain storage area is also monitored and controlled automatically. The occurrence of rats and pests in the storage area is controlled by ultrasound technique. SCADA software is used for real time monitoring of these processes.

\section{REFERENCES}

[1] "Programmable Logic Controllers", Frank .D. Petruzella.

[2] Supervisory Control And Data Acquisition (SCADA) basics, "http://en.wikipedia.org/wiki/SCADA".

[3] Electronic Pest Control, http://en.wikipedia.org/wiki/Electronic_pest_control".

[4] INA122 Instrumentation Amplifier data sheet.

[5] LM35 Temperature sensor data sheet.

[6] Gear motor working principle, "http://www.ehow.com/about-gear-motor". 
[7] Automated grain storage, "http://cornandsoybeandigest.com/grain-storage-autocontrol".

[8] Relay, "http://wiki.answers.com/Q/What is relay driver ULN2803".

\section{BIOGRAPHIES:}

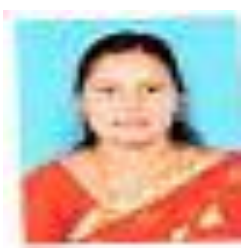

K. Kalaivani received the B.E degree in Electronics and communication engineering from Bharathiar university, Coimbatore,India in 1999. She received the M.E degree in Embedded systems from college of engineering, Guindy,Chennai, India in 2008. From 2001 to 2011 she was employed as a Assistant professor in Electronics and communication engineering department of R.M.K engineering college. In 2011 July onwards working as a Assistant Professor in Easwari engineering college. She is a research scholar doing Ph.D under the supervision of Dr.R.Sivakumar , R.M. K Engineering college.Her interest in research includes Multimedia Medical image processing and Neural networks. She is a member of IACSIT.

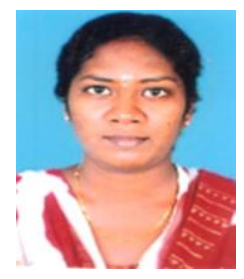

V.Anjalipriya received the B.E degree in Electronics and Instrumentation engineering from Anna University, Chennai, India in 2009. She received the M.E degree in Applied Electronics from SSN College of engineering, Chennai, India in 2011. From 2011 onwards working as a Assistant professor in Electronics and Instrumentation engineering department of Easwari engineering college. Her interest in research includes Bio medical Instrumentation. 